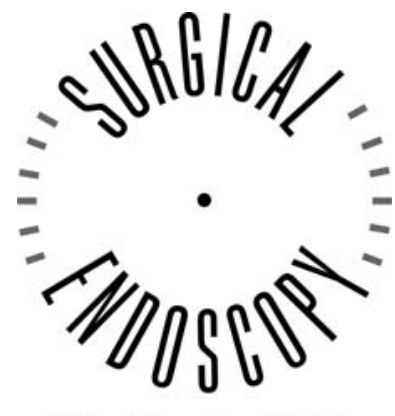

and Other Interventional Techniques

\title{
Postoperative hypoesthesia and pain: qualitative assessment after open and laparoscopic inguinal hernia repair
}

\author{
Guido Beldi, Non Haupt, Ramin Ipaktchi, Markus Wagner, Daniel Candinas \\ Department of Visceral and Transplantation Surgery, Inselspital Bern, 3010, Bern, Switzerland
}

Received: 5 February 2007/Accepted: 28 February 2007/Online publication: 19 August 2007

\begin{abstract}
Background: Chronic pain is an important outcome variable after inguinal hernia repair that is generally not assessed by objective methods. The aim of this study was to objectively investigate chronic pain and hypoesthesia after inguinal hernia repair using three types of operation: open suture, open mesh, and laparoscopic.

Methods: A total of 96 patients were included in the study with a median follow-up of 4.7 years. Open suture repair was performed in 40 patients (group A), open mesh repair in 20 patients (group B), and laparoscopic repair in 36 patients (group C). Hypoesthesia and pain were assessed using von Frey monofilaments. Quality of life was investigated with Short Form 36.

Results: Pain occurring at least once a week was found in $7(17.5 \%)$ patients of group A, in $5(25 \%)$ patients of group B, and in $6(16.6 \%)$ patients of group C. Area and intensity of hyposensibility were increased significantly after open nonmesh and mesh repair compared to those after laparoscopy $(p=0.01)$. Hyposensibility in patients who had laparoscopic hernia repair was significantly associated with postoperative pain $(p=0.03)$. Type of postoperative pain was somatic in 19 (61\%), neuropathic in 9 (29\%), and visceral in $3(10 \%)$ patients without significant differences between the three groups. Conclusions: The incidence of hypoesthesia in patients who had laparoscopic hernia repair is significantly lower than in those who had open hernia repair. Hypoesthesia after laparoscopic but not after open repair is significantly associated with postoperative pain. Von Frey monofilaments are important tools for the assessment of inguinal hypoesthesia and pain in patients who had inguinal hernia repair allowing quantitative and qualitative comparison between various surgical techniques.
\end{abstract}

This work was presented at the 10th World Congress of Endoscopic Surgery, Berlin, Germany, 13-16 September 2006

Correspondence to: Guido Beldi
Key words: Hernia — Pain — Hypoesthesia

With the introduction of prosthetic meshes, the recurrence rate of open and laparoscopic repair for inguinal hernia can be kept below 5\% [1, 2]. Long-term postoperative discomfort such as inguinal or scrotal pain and numbness has gained interest during the last years and has become one of the most important outcome variables besides recurrence rate $[3,4]$. Chronic pain occurs in up to $54 \%$ of patients after inguinal hernia repair and in $12 \%$ pain affects and impairs activities of daily life [5-8].

Current research focuses on technical aspects of inguinal hernia repair to decrease the incidence and severity of chronic pain from the different types. Generally, inguinal hernia can be treated by three approaches: open repair using suture or mesh and laparoscopic repair. Inguinal hernia repair using a mesh or laparoscopic inguinal hernia repair have been shown to have a lower the incidence of chronic pain $[5,9,8]$. However, as in most studies focusing on inguinal hernia repair, the quantification of pain was based on interviews of patients without objective assessment. Of 59 articles addressing chronic pain after inguinal hernia repair, only four examined some type of sensory function [5] and only one investigation used instruments for an objective assessment in open hernia repair [10].

To evaluate technical modifications and to consequently decrease the incidence of chronic pain, its pathomechanisms have to be investigated and understood in detail. Three types of chronic pain have been introduced by Poobalan et al. [8]: The most common type is somatic pain which is localized at the ligamentous insertion to the pubic tubercle and Coopers ligament and may be associated with tissue damage by suture or staple. Neuropathic pain develops in the sensory distribution of an injured nerve after direct damage to a nerve. The third pain syndrome is visceral pain from injury to somatic sacral or sympathetic nerves and is encountered upon ejaculation. 
Table 1. Patients characteristics

\begin{tabular}{llll}
\hline & Group A (open nonmesh) & Group B (open mesh) & Group C (laparoscopy) \\
\hline No. of patients & 40 & 20 & 36 \\
Male:Female & $38: 2$ & $20: 0$ & $31: 5$ \\
Age, median (range) & $61(28-77)$ & $62(45-83)$ & $57(28-70)$ \\
Operative time, median (range) & $78(40-120)$ & $68(40-125)$ & $66(25-150)$ \\
General anesthesia & 22 & 13 & 36 \\
Regional anesthesia & 17 & 7 & 0 \\
Local anesthesia & 1 & 0 & 0 \\
Type of hernia & 15 & 12 & 18 \\
Direct & 19 & 6 & 25 \\
Indirect & 10 & 4 & 7 \\
Combined & 1 & 1 & 0 \\
Femoral & 4 & 4 & 9 \\
Recurrent hernia & 5 & 3 & 14 \\
Bilateral & 45 & 23 & 50 \\
Number of hernias & & 7 & \\
\hline
\end{tabular}

A tool for objective measurement of pain and hypoesthesia seems needed to define and describe postoperative pain and to test and compare modifications of various surgical techniques. The aim of this study was to objectively assess chronic hypoesthesia and pain after inguinal hernia repair using von Frey monofilaments and to compare open suture, open mesh, and laparoscopic techniques.

\section{Materials and methods}

Patients with a minimal follow-up of 12 months were included in this study. Between January 1995 and November 2003 a total of 316 patients underwent inguinal hernia repair in our institution. Patients were grouped according to the type of hernia repair: group A: open suture repair, group B: open mesh repair, and group C: laparoscopic mesh repair. The indication for the technique depended on the surgeon's preference. Demographic and clinical data of these patients are summarized in Table 1. Approval from the regional ethics committee was obtained.

Postoperative clinical evaluation was obtained during an interview by an independent observer. The observer assessing the data has neither operated nor assisted in any of the operations and was not involved in the postoperative treatment of the patients.

The International Association for the Study of Pain definition of chronic pain (pain persisting beyond the normal tissue healing time assumed to be three months) was used as the primary outcome measure [11]. For the description of chronic pain three criteria have been assessed: character, quality, and location of pain. The character of pain was somatic, visceral,or neuropathic depending on the presence of numbness and sensations.

All patients obtained a standard questionnaire containing a visual analog scale (VAS, 0-10) to assess pain intensity and the Short Form 36 (SF-36) to assess quality of life. A body chart was included in the questionnaire on which participants could mark the location of pain and use symbols to indicate its characteristics. Questionnaires were filled in before clinical examination.

For quantitative assessment of postoperative pain and hypoesthesia we used calibrated von Frey monofilaments (Senselab Aesthesiometer, Somedic, Stockholm, Sweden). With the monofilaments a defined pressure is applied to the region of interest (No. 4: $0.5 \mathrm{mN}$, No. 5: $0.8 \mathrm{mN}$, No. $6: 1 \mathrm{mN}$, No. 7: $2 \mathrm{mN}$, No. $8: 3 \mathrm{mN}$, No. 9: $6 \mathrm{mN}$, No. 10: $12 \mathrm{mN}$, No. 11: $22 \mathrm{mN}$, No. 12: $34 \mathrm{mN}$, No. 13: $58 \mathrm{mN}$, No. 14: 89 $\mathrm{mN}$, No. 15: $212 \mathrm{mN}$, No. 16: $306 \mathrm{mN}$, No. 17: $468 \mathrm{mN}$ ). The mechanical pain threshold was defined as the lowest force that elicited a sensation of pain or discomfort. The threshold for hypoesthesia was defined as the least pressure eliciting any sensation. Computer-generated graphs were plotted. The score for hypoesthesia was calculated by adding intensity values of hypoesthesia as measured by von Frey monofilaments.

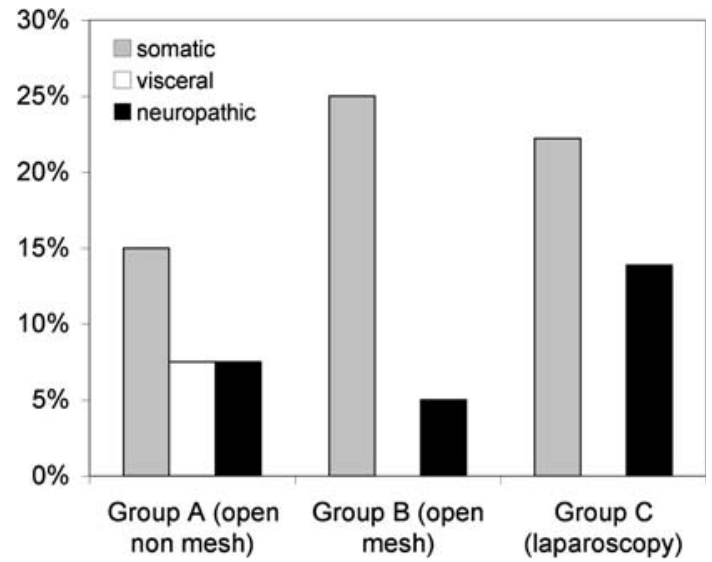

Fig. 1. Percentage of patients with somatic, visceral, and neuropathic pain.

Surgical technique. Prophylactic antibiotics were used in all groups. The open nonmesh operation was performed as a modified two-layer Shouldice repair 2-0 using a polypropylene suture [12]. For the open mesh repair a standard Lichtenstein procedure with a polypropylene mesh (Prolene, Ethicon Schweiz, Johnson \& Johnson Medical, CH-8957 Spreitenbach) was performed [13]. For laparoscopic hernia repair a transperitoneal or preperitoneal approach was used [14].

The statistical analysis was carried out using SPSS for Windows (SPSS Inc., Chicago IL, USA). The SF-36 questionnaire was analyzed using the Kruskal-Wallis test. Responses to the SF-36 were summed to yield eight scores, each ranging from 0 (worst health possible) to 100 (best health possible). Proportions were compared using Fisher's exact test and the chi-squared test; $p<0.05$ was considered statistically significant.

\section{Results}

A total of 96 patients who underwent clinical examination and completed questionnaires were included in the study. Group A had 40 patients with 45 hernias, group $\mathrm{B}$ had 20 patients with 23 hernias, and group $\mathrm{C}$ had 36 patients with 50 hernias. Patients' characteristics are outlined in Table 1.

Mean follow-up in group A was 5.2 years (standard deviation $[\mathrm{SD}]=1.48$ ), in group $\mathrm{B}$ it was 2.4 years 

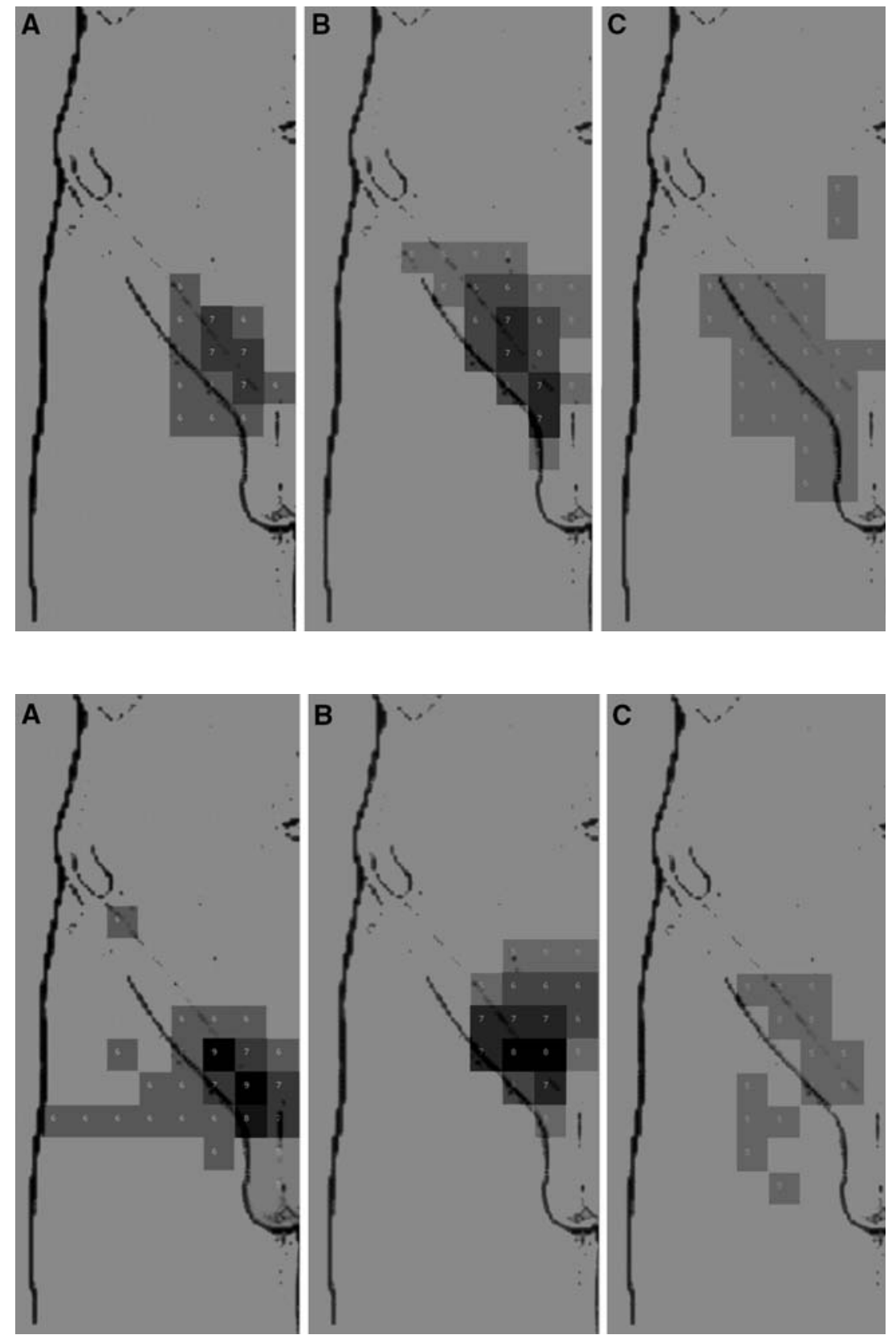

Fig. 2. Localization of hyposensibility in patients with (A) open nonmesh, (B) open mesh, and (C) laparoscopic hernia repair.
Fig. 3. Localization of hyposensibility in patients with pain after (A) open nonmesh, (B) open mesh, and $(\mathbf{C})$ laparoscopic hernia repair.
$(\mathrm{SD}=1.53)$, and in group $\mathrm{C}$ it was 6.3 years $(\mathrm{SD}=$ 1.99). At the follow-up examination recurrent hernia was found once in each group. Overall postoperative pain was encountered in $12(26.7 \%)$ of group A, in $6(26.1 \%)$ of group B, and in $13(26.0 \%)$ of group C. Mean pain values of these patients measured with the VAS were 1.8 $(\mathrm{SD}=1.8)$ in group $\mathrm{A}, 2.7(\mathrm{SD}=2.7)$ in group $\mathrm{B}$, and $1.9(\mathrm{SD}=1.6)$ in group C. Pain occurring at least once a week was found in $7(17.5 \%)$ patients of group A, in 5 (25\%) patients of group B, and in $6(16.6 \%)$ patients of group $\mathrm{C}$. There was no significant difference between the three groups. The relative incidence of somatic, visceral, and neuropathic pain of all patients with postoperative pain is displayed in Figure 1 [8].

Results of the SF-36 are displayed in Table 2. The only significant difference was found in general health.
Patients of group A had a significantly higher estimate of their general health.

Hypoesthesia was appreciated by $67 \%$ of the patients who had open nonmesh surgery, by $65 \%$ patients who had open mesh surgery, and by $54 \%$ who had laparoscopic surgery. Hypoesthesia in the sensory region of the ilioinguinal and genitofemoral nerves was observed in 52\% of the patients who had open nonmesh surgery, in $45 \%$ of the patients who had open mesh surgery, and in $42.5 \%$ who had laparoscopic surgery. Localization of hypoesthesia is shown in Figure 2. In both groups of patients with open hernia repair, hypoesthesia was localized in proximity of the inguinal ligament. The region of hypoesthesia in patients with open repair using mesh was localized more laterally than without the use of mesh. The median hypoesthesia score in group A was 56 (range $=0-198$ ), in 
Table 2. Quality-of-life assessment by Short Form 36 (SF-36)

\begin{tabular}{|c|c|c|c|c|c|c|}
\hline & \multicolumn{2}{|c|}{ Group A (open nonmesh) } & \multicolumn{2}{|c|}{ Group B (open mesh) } & \multicolumn{2}{|c|}{ Group C (laparoscopy) } \\
\hline & Median & Range & Median & Range & Median & Range \\
\hline Physical function & 95 & $70-100$ & 94.7 & $87.5-100$ & 97.5 & $90-100$ \\
\hline Role physical & 100 & $100-100$ & 100 & $100-100$ & 100 & $100-100$ \\
\hline Bodily pain & 0 & $0-35$ & 0 & $0-0$ & 0 & $0-5$ \\
\hline General health & 55 & $42.5-62.5$ & 43.8 & $40-52.5$ & 45 & $40-50$ \\
\hline Vitality & 50 & $45-55$ & 50 & $45-52.5$ & 45 & $42.5-55$ \\
\hline Social function & 50 & $50-50$ & 50 & $50-50$ & 50 & $50-50$ \\
\hline Role emotional & 100 & $83.3-100$ & 100 & $100-100$ & 100 & $100-100$ \\
\hline Mental health & 60 & $56-64$ & 60 & $54.7-64$ & 64 & $56-64$ \\
\hline
\end{tabular}

Table 3. Hypoesthesia in patients with and without chronic pain

\begin{tabular}{|c|c|c|c|c|c|c|c|}
\hline & \multicolumn{2}{|c|}{ Group A (open nonmesh) } & \multicolumn{2}{|c|}{ Group B (open mesh) } & \multicolumn{2}{|c|}{ Group C (laparoscopy) } & \multirow[b]{2}{*}{$p$ Value $^{\mathrm{a}}$} \\
\hline & Median & Range & Median & Range & Median & Range & \\
\hline Overall & 56 & $0-198$ & 59 & $0-231$ & 9 & $0-506$ & 0.01 \\
\hline No pain & 49 & $0-198$ & 53 & $0-231$ & $0^{\mathrm{b}}$ & $0-506$ & 0.03 \\
\hline Chronic pain & 71 & $0-165$ & 63 & $0-150$ & $24^{\mathrm{b}}$ & $0-72$ & 0.07 \\
\hline
\end{tabular}

${ }^{a}$ Kruskal-Wallis test. The difference between patients without pain and patients with chronic pain was significantly different after laparoscopic hernia repair ( $p=0.03$, Mann-Whitney test).

group B 59 (0-231), and in group C 9 (0-506) (Table 3). In contrast with open repair, the region of hypoesthesia after laparoscopic inguinal hernia repair was significantly decreased and is less localized to the groin region. Its localization tends to reach further distal and proximal of the inguinal ligament.

Allodynia was seen in two patients: one open mesh implantation and one laparoscopic hernia repair.

Localization of hypoesthesia of the patients with chronic postoperative pain is shown in Figure 3. Localization and intensity of hypoesthesia and pain did not vary between open mesh and suture repair. In patients who had open hernia repair, pain was localized in the proximity of the pubic tubercle and inguinal ligament. In laparoscopic repair the distribution of hypoesthesia covered a larger area and was less well defined to the inguinal ligament than in open repair.

\section{Discussion}

Objective assessment of inguinal sensations after inguinal hernia repair revealed differences in type and localization of pain and hypoesthesia between three different types of hernia repair. Hypoesthesia in laparoscopic surgery was less localized near the inguinal ligament and less intense than in open surgery, showing the importance of the inguinal skin incision for the development of a well-defined area of hypoesthesia distal of the incision. However, if patients who had laparoscopic hernia repair develop hypoesthesia, it is significantly associated with postoperative pain unlike in patients after open surgery. Hypoesthesia in the groin of patients who had laparoscopic hernia repair may be the result of lesions of the subcostal, iliohypogastric, ilioinguinal, and genitofemoral nerves.
The overall incidence of chronic postoperative pain after open suture, open mesh, and laparoscopic inguinal hernia repair is $26.3 \%$. Overall, no difference was found among the three groups. Our findings mirror the results of recent studies that compared open mesh and open suture repair in which no difference was found between the two groups [15-17]. Comparing open and laparoscopic hernia repair, the incidence in large-scale studies showed a reduction of chronic pain after laparoscopic repair [18-20]. However, these studies lacked objective pain assessment and descriptions of the type of pain.

Objective measurements of postoperative inguinal sensations are important for current and future investigations, especially in studies that focus on aspects of the surgical technique and implanted materials. Absorbable tissue adhesives for mesh fixation are currently compared with standard suture fixation in open and laparoscopic surgery [21-24]. Other modifications have to be monitored to reduce long-term discomfort. Objective assessment of pain and hypoesthesia by von Frey monofilaments prior to and after surgery will allow one to address individual surgical factors precisely.

Acknowledgments. The authors thank Ramun Berger for the generation of a computer model to calculate and display measured data by von Frey monofilaments.

\section{References}

1. Grant AM (2002) Open mesh versus non-mesh repair of groin hernia: meta-analysis of randomised trials based on individual patient data [corrected]. Hernia 6(3): 130-136 
2. Memon MA, Cooper NJ, Memon B, Memon MI, Abrams KR (2003) Meta-analysis of randomized clinical trials comparing open and laparoscopic inguinal hernia repair. Br J Surg 90(12): 1479-1492

3. Bay-Nielsen M, Perkins FM, Kehlet H (2001) Pain and functional impairment 1 year after inguinal herniorrhaphy: a nationwide questionnaire study. Ann Surg 233(1): 1-7

4. Poobalan AS, Bruce J, King PM, Chambers WA, Krukowski ZH, Smith WC (2001) Chronic pain and quality of life following open inguinal hernia repair. Br J Surg 88(8): 1122-1126

5. Aasvang E, Kehlet H (2005) Chronic postoperative pain: the case of inguinal herniorrhaphy. Br J Anaesth 95(1): 69-76

6. Callesen T, Bech K, Kehlet H (1999) Prospective study of chronic pain after groin hernia repair. Br J Surg 86(12): 1528-1531

7. Cunningham J, Temple WJ, Mitchell P, Nixon JA, Preshaw RM, Hagen NA (1996) Cooperative hernia study. Pain in the postrepair patient. Ann Surg 224(5): 598-602

8. Poobalan AS, Bruce J, Smith WC, King PM, Krukowski ZH, Chambers WA (2003) A review of chronic pain after inguinal herniorrhaphy. Clin J Pain 19(1): 48-54

9. McCormack K, Scott NW, Go PM, Ross S, Grant AM (2003) Laparoscopic techniques versus open techniques for inguinal hernia repair. Cochrane Database Syst Rev (1):CD001785

10. Mikkelsen T, Werner MU, Lassen B, Kehlet H (2004) Pain and sensory dysfunction 6 to 12 months after inguinal herniotomy. Anesth Analg 99(1): 146-151

11. International Association for the Study of Pain (1986) Classification of chronic pain. Descriptions of chronic pain syndromes and definitions of pain terms. Prepared by the International Association for the Study of Pain Subcommittee on Taxonomy. Pain Suppl 3: S1-S226

12. Striffeler H, Zufferey S, Schweizer W (1993) Quality control after introduction of a new hernia technique. Barwell transversal fasciaplasty. Helv Chir Acta 59(5-6): 771-774

13. Amid PK, Shulman AG, Lichtenstein IL (1996) Open "tensionfree" repair of inguinal hernias: the Lichtenstein technique. Eur J Surg 162(6): 447-453
14. Gerber S, Hammerli PA, Glattli A (2000) Laparoscopic transabdominal preperitoneal hernioplasty. Evaluation of complications due to transabdominal approach. Chirurg 71(7): 824-828

15. Bay-Nielsen M, Nilsson E, Nordin P, Kehlet H (2004) Chronic pain after open mesh and sutured repair of indirect inguinal hernia in young males. Br J Surg 91(10): 1372-1376

16. Nordin P, Bartelmess P, Jansson C, Svensson C, Edlund G (2002) Randomized trial of Lichtenstein versus Shouldice hernia repair in general surgical practice. Br J Surg 89(1): 45-49

17. Vrijland WW, van den Tol MP, Luijendijk RW, Hop WC, Busschbach JJ, de Lange DC, van Geldere D, Rottier AB, Vegt PA, JN IJ, Jeekel J (2002) Randomized clinical trial of nonmesh versus mesh repair of primary inguinal hernia. Br J Surg 89(3): 293-297

18. MRC Laparoscopic Groin Hernia Trial Group (1999) Laparoscopic versus open repair of groin hernia: a randomised comparison. The MRC Laparoscopic Groin Hernia Trial Group. Lancet 354(9174): 185-190

19. EU Hernia Trialists Collaboration (2002) Repair of groin hernia with synthetic mesh: meta-analysis of randomized controlled trials. Ann Surg 235(3): 322-332

20. Kumar S, Wilson RG, Nixon SJ, Macintyre IM (2002) Chronic pain after laparoscopic and open mesh repair of groin hernia. $\mathrm{Br} \mathbf{J}$ Surg 89(11): 1476-1479

21. Canonico S, Santoriello A, Campitiello F, Fattopace A, Corte AD, Sordelli I, Benevento R (2005) Mesh fixation with human fibrin glue (Tissucol) in open tension-free inguinal hernia repair: a preliminary report. Hernia 9: 330-333

22. Helbling C, Schlumpf R (2003) Sutureless Lichtenstein: first results of a prospective randomised clinical trial. Hernia 7(2): 80-84

23. Hidalgo M, Castillo MJ, Eymar JL, Hidalgo A (2005) Lichtenstein inguinal hernioplasty: sutures versus glue. Hernia 9: 242-244

24. Topart P, Vandenbroucke F, Lozac'h P (2005) Tisseel versus tack staples as mesh fixation in totally extraperitoneal laparoscopic repair of groin hernias: a retrospective analysis. Surg Endosc 19(5): 724-727 